\title{
A WIENER INVERSION-TYPE THEOREM
}

\author{
JAMES R. HOLUB
}

\begin{abstract}
Let $W(D)=\left\{f(z)=\sum_{n=0}^{\infty} a_{n} z^{n}\left|\|f\|_{1}=\sum_{n=0}^{\infty}\right| a_{n} \mid<+\infty\right\}$, $f(z)$ a function in $W(D)$ for which $f(0)=1$, and $M_{f}$ the operator of multiplication by $f(z)$ on $W(D)$. It is shown that if $k$ and $m$ are integers for which $0 \leq m \leq k-1$ and $X_{k}^{m}$ is the closed subspace of $W(D)$ spanned by $\left\{z^{n k+i} \mid n=0,1, \ldots ; i=0,1, \ldots, m\right\}$, then $M_{f}$ is bounded below on $X_{k}^{m} \Leftrightarrow f(z)$ does not have $k-m$ distinct zeros in any set of the form $\left\{w^{i} z_{0}|0 \leq i \leq k-1 ;| z_{0} \mid=1\right\}$, where $w$ is a primitive kth root of unity.
\end{abstract}

1. Let $W(D)$ denote the Wiener disc algebra consisting of those analytic functions $f(z)=\sum_{n=0}^{\infty} a_{n} z^{n}$ for which $\|f\|_{1}=\sum_{n=0}^{\infty}\left|a_{n}\right|<+\infty$. The classical Wiener inversion theorem states that if $f(\theta)=\sum_{n=-\infty}^{\infty} a_{n} e^{i n \theta}$, where $\sum_{n=-\infty}^{\infty}\left|a_{n}\right|<+\infty$, then $1 / f(\theta)=\sum_{n=-\infty}^{\infty} b_{n} e^{i n \theta}$ with $\sum_{n=-\infty}^{\infty}\left|b_{n}\right|<+\infty \Leftrightarrow f(\theta) \neq 0$ for all $\theta[\mathbf{3}$, p. 196]. A simple corollary is that if $f(z) \in W(D)$ then multiplication by $f(z)$ on $W(D)$ is an operator $M_{f}$ which is bounded below $\Leftrightarrow f(z) \neq 0$ for all $|z|=1$. Consequently, a sequence in $W(D)$ of the form $\left\{z^{n} f(z)\right\}_{n=0}^{\infty}$ is a basic sequence which is equivalent to the basis $\left\{z^{n}\right\}_{n=0}^{\infty}$ for $W(D) \Leftrightarrow f(z) \neq 0$ for all $|z|=1[\mathbf{1}]$.

In [2] a result of considerably greater generality was proved. In the context of multiplication operators this result says that if $f(z) \in W(D)$ and $k$ is an integer $\geq 1$ then the operator $M_{f}$ of multiplication by $f(z)$ is bounded below on the closed subspace $\left[z^{n k}\right]_{n=0}^{\infty}$ of $W(D) \Leftrightarrow f(z)$ has no set of $k$ zeros of the form $\left\{w^{i} z_{0}\right\}_{i=0}^{k-1}$, where $\left|z_{0}\right|=1$ and $w$ is a primitive kth root of unity.

The purpose of this paper is not only to prove a still more general inversion theorem of this type, but to unify the results of [1 and 2] mentioned above by fitting them into a context in which they occur as the natural extreme cases of a general theory relating invertibility of $M_{f}$ to the number of "cyclic zeros" of $f(z)$ on the circle $|z|=1$. As a corollary we derive a characterization of certain cyclically shifted sequences in $W(D)$ which are basic and equivalent to the basis $\left\{z^{n}\right\}_{n=0}^{\infty}$, thereby completing work begun in [1 and 2].

2. Throughout the paper $f(z)$ will denote a function in $W(D)$ for which $f(0)=$ $1, k$ will be a fixed integer $\geq 1$, and $w$ a primitive $k$ th root of unity.

If $m$ is an integer for which $0 \leq m \leq k-1$ let $X_{k}^{m}$ denote the closed subspace of $W(D)$ spanned by the sequence $\left\{z^{n k+i} \mid n=0,1,2, \ldots ; i=0,1, \ldots, m\right\}$. Then for any $k \geq 1, X_{k}^{0}=\left[z^{n k}\right]_{n=0}^{\infty}, X_{k}^{k-1}=W(D)$, and $\left\{X_{k}^{m}\right\}_{m=0}^{k-1}$ is an increasing tower of subspaces of $W(D)$ between these two "endpoints". Viewed from this perspective the following theorem simultaneously generalizes and unifies the results of $[\mathbf{1}$ and

Received by the editors July 11, 1985.

1980 Mathematics Subject Classification. Primary 47B37, 47B38, 46B15; Secondary 47A05, $46 \mathrm{E} 15$.

Key words and phrases. Wiener disc algebra, multiplication operator, inversion theorem, basic sequence, shift basic sequence. 
2] by describing the invertibility of $M_{f}$ on the spaces $X_{k}^{m}$ in terms of the number of zeros of $f(z)$ lying in sets of the form $\left\{w^{k} z_{0}\right\}_{i=0}^{k-1}$ on the unit circle. Of particular interest is the way in which this dependence is a monotonic function of $m$.

THEOREM. The operator $M_{f}$ is bounded below on $X_{k}^{m} \Leftrightarrow f(z)$ does not have $k-m$ distinct zeros in any set of the form $\left\{w^{i} z_{0}|0 \leq i \leq k-1,| z_{0} \mid=1\right\}$.

Proof. Suppose $M_{f}$ is not bounded below on $X_{k}^{m}$. Then there is a sequence $\left\{g_{j}(z)\right\}_{j=1}^{\infty}$ in $X_{k}^{m}$ for which $\left\|g_{j}\right\|_{1}=1$ for all $j$, but for which $\left\|f(z) g_{j}(z)\right\|_{1} \rightarrow 0$. By definition of the space $X_{k}^{m}$ each $g_{j}(z)$ can be written in the form $g_{j}(z)=$ $\sum_{i=0}^{m} z^{i} h_{i}^{(j)}(z)$, where $h_{i}^{(j)}(z) \in\left[z^{n k}\right]_{n=0}^{\infty}$ for all $i$ and $j$, and for which $\sum_{i=0}^{m}\left\|h_{i}^{(j)}\right\|_{1}$ $=\left\|g_{j}\right\|_{1}=1$ for all $j$. Since $h_{i}^{(j)}(z) \in\left[z^{n k}\right]_{n=0}^{\infty}$, we have $h_{i}^{(j)}\left(w^{p} z\right)=h_{i}^{(j)}(z)$ for all integers $p$, while from the condition $\sum_{i=0}^{m}\left\|h_{i}^{(j)}\right\|_{1}=1$ we may assume (taking a subsequence of $\left\{g_{j}\right\}$ if necessary) that $\max _{0 \leq i \leq m} \inf _{j}\left\|h_{i}^{(j)}\right\|_{1}>0$. Therefore, setting $f(z) \cdot g_{j}(z)=q_{j}(z)$ for all $j$ and using the observations above together with the assumption that $q_{j} \rightarrow 0$ as $j \rightarrow \infty$ we have that

$$
\left(f\left(w^{l} z\right)\right)\left(\sum_{i=0}^{m} w^{l i} z^{i} h_{i}^{(j)}(z)\right)=q_{j}\left(w^{l} z\right) \stackrel{j}{\rightarrow} 0 \quad \text { for all } l=0,1,2, \ldots, m .
$$

Hence

$$
\begin{gathered}
\left(\prod_{i=0}^{m} f\left(w^{i} z\right)\right)\left[\begin{array}{cccc}
1 & 1 & \cdots & 1 \\
1 & w & \cdots & w^{m} \\
1 & w^{2} & \cdots & w^{2 m} \\
1 & w^{m} & \cdots & w^{m^{2}}
\end{array}\right]\left[\begin{array}{c}
h_{0}^{(j)}(z) \\
z h_{1}^{(j)}(z) \\
z^{m} h_{m}^{(j)}(z)
\end{array}\right] \\
=\left[\begin{array}{c}
\prod_{i \neq 0} f\left(w^{i} z\right) \cdot q_{j}(z) \\
\prod_{i \neq 1} f\left(w^{i} z\right) \cdot q_{j}(w z) \\
\prod_{i \neq m} f\left(w^{i} z\right) \cdot q_{j}\left(w^{m} z\right)
\end{array}\right] \stackrel{j}{\rightarrow}\left[\begin{array}{l}
0 \\
0 \\
0
\end{array}\right] .
\end{gathered}
$$

Now since $1, w, w^{2}, \ldots, w^{m}$ are distinct, the matrix

$$
\left[\begin{array}{cccc}
1 & 1 & \cdots & 1 \\
1 & w & \cdots & w^{m} \\
1 & w^{m} & \cdots & w^{m^{2}}
\end{array}\right]
$$

has a nonzero (Vandermonde) determinant, and hence is invertible. It follows that

$$
\left(\prod_{i=0}^{m} f\left(w^{i} z\right)\right)\left[\begin{array}{c}
h_{0}^{(j)}(z) \\
z h_{1}^{(j)}(z) \\
\vdots \\
z^{m} h_{m}^{(j)}(z)
\end{array}\right] \stackrel{j}{\rightarrow}\left[\begin{array}{c}
0 \\
0 \\
\vdots \\
0
\end{array}\right],
$$

and hence that the sequence $\left\{\left(\prod_{i=0}^{m} f\left(w^{i} z\right)\right) \cdot\left(h_{l}^{(j)}(z)\right)\right\}_{j=1}^{\infty}$ converges to zero in $W(D)$ for each $l=0,1, \ldots, m$.

In exactly the same way, if $\sigma$ is any subset of $\{0,1,2, \ldots, k-1\}$ with cardinality $m+1$ then since $\left\{w^{i}\right\}_{i \in \sigma}$ is again a set of distinct numbers, we have $\left(\prod_{i \in \sigma} f\left(w^{i} z\right)\right)\left(h_{l}^{(j)}(z)\right) \stackrel{j}{\rightarrow} 0$ in $W(D)$ for each $l=0,1, \ldots, m$, where, for some $l$, 
$\inf _{j}\left\|h_{l}^{(j)}\right\|_{1}>0$ according to our earlier remarks. Thus $\left\{\prod_{i \in \sigma} f\left(w^{i} z\right) \mid \operatorname{card} \sigma=\right.$ $m+1\}$ has a common zero $z_{0}$ on the unit circle. For, if $A=\{f(\theta) \mid f(\theta)=$ $\sum_{n=-\infty}^{\infty} a_{n} e^{-i n \theta}$, with $\left.\|f\|=\sum_{n=-\infty}^{\infty}\left|a_{n}\right|<+\infty\right\}$, then we can identify $W(D)$ with the subspace of $A$ consisting of all such $f(\theta)$ with $a_{n}=0$ for $n<0$. The proper maximal ideals in $A$ are sets of the form $\left\{f(\theta) \mid f\left(\theta_{0}\right)=0\right\}$ for some $\theta_{0} \in[0,2 \pi][3$, p. 195], so if $\left\{\prod_{i \in \sigma} f\left(w^{i} z\right) \mid \operatorname{card} \sigma=m+1\right\}$ did not have a common zero on the unit circle it would follow that the corresponding maximal ideal in $A$ generated by this set must be all of $A$. Consequently, there would exist functions $\left\{r_{\sigma}(\theta)\right\}$ in $A$ for which $1=\sum_{\sigma} r_{\sigma}(\theta)\left(\prod_{k \in \sigma} f\left(w^{k} e^{i \theta}\right)\right)$, and hence for which

$$
h_{l}^{(j)}\left(e^{i \theta}\right)=\sum_{\sigma} r_{\sigma}(\theta) \prod_{k \in \sigma} f\left(w^{k} e^{i \theta}\right) h_{l}^{(j)}\left(e^{i \theta}\right)
$$

for all $l$ and $j$. But by the remarks above this is impossible since the latter sum converges to zero in $A$ as $j \rightarrow \infty$, yet $\inf _{j}\left\|h_{l}^{(j)}\right\|_{1}>0$. Hence we conclude there must be a number $z_{0}$ with $\left|z_{0}\right|=1$ for which $\prod_{i \in \sigma} f\left(w^{i} z_{0}\right)=0$ for all subsets $\sigma$ of $\{0,1, \ldots, k-1\}$ of cardinality $m+1$. But then $f\left(w^{i} z_{0}\right) \neq 0$ for at most $m$ values of $i$, where $0 \leq i \leq k-1$, so $f\left(w^{i} z_{0}\right)=0$ for at least $k-m$ such values of $i$, and we see that $f(z)$ has $k-m$ zeros in the set $\left\{w^{i} z_{0}\right\}_{i=0}^{k-1}$.

On the other hand, suppose that $f\left(w^{i} z_{0}\right)=0$ for some $\left|z_{0}\right|=1$ and for all $i$ in a subset $\sigma_{0}$ of $\{0,1, \ldots, k-1\}$ of cardinality $k-m$. In particular, suppose $f(z)=\prod_{i \in \sigma_{0}}\left(1-z / w^{i} z_{0}\right)$ (the general case reduces easily to this one, as we show later). Letting $\sigma_{0}^{\prime}=\{0,1, \ldots, k-1\} \backslash \sigma_{0}$ we have the cardinality of $\sigma_{0}^{\prime}=m$, while

$$
1 / f(z)=\frac{\prod_{i \in \sigma_{0}^{\prime}}\left(1-z / w^{i} z_{0}\right)}{1-z^{k} / z_{0}^{k}}=\left[\prod_{i \in \sigma_{0}^{\prime}}\left(1-\frac{z}{w^{i} z_{0}}\right)\right]\left[\sum_{n=0}^{\infty} \frac{z^{n k}}{z_{0}^{n k}}\right]=\sum_{j=0}^{\infty} b_{j} z^{j}
$$

where

(i) $\left|b_{j}\right|=\left|b_{n k+j}\right|$ for all $j$ and all $n$ (since $\left|z_{0}\right|=1$ ), and

(ii) $b_{j}=0$ for $j \notin\{n k+i \mid n=0,1, \ldots ; i=0,1, \ldots, m\}$ (since the degree of $\prod_{i \in \sigma_{0}^{\prime}}\left(1-z / w^{i} z_{0}\right)$ is equal to $\left.m\right)$.

By (ii), for any $r \geq 0$ the polynomial $\sum_{j=0}^{r} b_{j} z^{j} \in X_{k}^{m}$, while from (i) it follows that for any $\varepsilon>0$ there is a constant $c$ and an integer $r$ for which $\left\|\sum_{i=0}^{r} c b_{j} z^{j}\right\|_{1}=1$ and $\sup _{0 \leq j \leq r}\left|c b_{j}\right|<\varepsilon$. Since $f(z)$ is a polynomial of degree $k-m$ and $1 / f(z)=$ $\sum_{j=0}^{\infty} b_{j} z^{j}$, for any $r$ the function $f(z) \cdot \sum_{j=0}^{r} b_{j} z^{j}$ is equal to $1+z^{r+1} q_{r}(z)$, where $q_{r}(z)$ is some polynomial of degree $k-m-1$ whose coefficients (for any $r$ ) are bounded by $L=\sup _{j}\left|b_{j}\right| \cdot\|f(z)\|_{1}<+\infty$. Hence, by the above, for any $\varepsilon>0$ we can pick $|c|<\varepsilon$ and some $r$ so that $\left\|\sum_{j=0}^{r} c b_{j} z^{j}\right\|_{1}=1$, but for which $\sup _{j}|c|\left|b_{j}\right|<\varepsilon$, and hence for which

$$
\begin{aligned}
\left\|(f(z))\left(\sum_{j=0}^{r} c b_{j} z^{j}\right)\right\|_{1} & \leq|c|\left\|1+z^{r+1} q_{r}(z)\right\|_{1} \\
& \leq|c|\left[1+(k-m) \sup _{j}\left|b_{j}\right|\|f(z)\|_{1}\right] \\
& \leq|c|+(k-m) \cdot \varepsilon \cdot\|f(z)\|_{1}<\varepsilon\left[1+(k-m)\|f(z)\|_{1}\right] .
\end{aligned}
$$

That is, $M_{f}$ is not bounded below on $X_{k}^{m}$. 
For the general case, suppose $f(z)$ is an arbitrary function in $W(D)$ for which $f\left(w^{i} z_{0}\right)=0$ for some $\left|z_{0}\right|=1$ and for all $i \in \sigma_{0}$, a subset of $\{0,1, \ldots, k-1\}$ with cardinality $k-m$. Since the polynomials are dense in $W(D)$ and the subspace $X=\left\{g(z) \in W(D) \mid g\left(w^{i} z_{0}\right)=0, i \in \sigma_{0}\right\}$ is of finite codimension in $W(D)$, the polynomials in $X$ are also dense in $X$. It follows that $f(z)$ can be approximated as closely as desired by a polynomial of the form $q(z)=p_{\sigma_{0}}(z) r(z)$, where $p_{\sigma_{0}}(z)=$ $\prod_{i \in \sigma}\left(1-z / w^{i} z_{0}\right)$ (and $r(z)$ is some other polynomial). Hence if $M_{f}$ were bounded below on $X_{k}^{m}$ it would follow that $M_{q}$ would also be bounded below on $X_{k}^{m}$ for some such $q(z)$, an impossibility since we just showed that $M_{p_{\sigma_{0}}}$ is not bounded below on $X_{k}^{m}$. Thus if $f(z)$ has $k-m$ zeros in the set $\left\{w^{i} z_{0}|0 \leq i \leq k-1,| z_{0} \mid=1\right\}$ then $M_{f}$ cannot be bounded below on $X_{k}^{m}$, and the proof is complete.

From this theorem we get the following result which incorporates those of [ 1 and 2] into a unified description of cyclically shifted basic sequences in $W(D)$.

COROLLARY. If $f(z) \in W(D)$ with $f(0)=1, k$ is an integer, and $m$ is an integer for which $0 \leq m \leq k-1$, then a sequence in $W(D)$ of the form $\left\{z^{k n+i} f(z) \mid n=\right.$ $0,1, \ldots ; i=0,1, \ldots, m\}$ is a basic sequence equivalent to the basis $\left\{z^{n}\right\}_{n=0}^{\infty}$ for $W(D) \Leftrightarrow f(z)$ does not have $k-m$ distinct zeros in any set of the form $\left\{w^{i} z_{0} \mid 0 \leq\right.$ $\left.i \leq k-1,\left|z_{0}\right|=1\right\}$.

\section{REFERENCES}

1. J. Holub, On bases and the shift operator, Studia Math. 71 (1981), 191-202.

2. Shift basic sequences in the Wiener disc algebra, Proc. Amer. Math. Soc. 88 (1983), 464-468.

3. M. Naimark, Normed rings, Noordhoff, Groningen, 1964.

Department of Mathematics, Virginia Polytechnic Institute and State UNIVERSITY, BLACKSBURG, VIRGINIA 24060 